\title{
Zonificación de las áreas propensas a incidentes por ataques de Crocodylus acutus en el Pacífico Central de Costa Rica utilizando un Sistema de Información Geográfico
}

\author{
Luis Fernando Sandoval Murillo* \\ Carlos Morera Beita* \\ Iván Sandoval Hernández**
}

Recibido 9 de octubre de 2018; aceptado 20 de diciembre de 2018

\begin{abstract}
In recent years, the incidence of attacks by crocodiles in Costa Rica has increased, which is associated with the development of human activities in areas considered as optimal in what I determine as the species' potential habitat. Given the above, the present investigation proposes the identification of zones of interaction between the crocodile and the human being from the integration of physical-geographic variables through Geographic Information Systems (GIS). In this way, a theoretical compilation was carried out to identify the physical-geographical variables that make up the crocodile's habitat (slope, drainage network, flood areas and land use coverage), as well as the socio-environmental variables that influence the alteration and loss of habitat of the species (number of neighborhoods, population density, density of roads, and coverage of land use). Likewise, case records were integrated with crocodiles assisted by the fire department. It was possible to demonstrate that most of incidents occur in areas with high interaction (areas where a wide variety of anthropic activities are carried out within the potential habitat of this reptile.) In addition, the results obtained in the investigation constitute an important input in the decision-making at the policy and territorial order level in the Central Pacific Region, since tourism is one of the main economic activities that can be threatened by an attack by this species.

* Académico, Escuela de Ciencias Geográficas, Universidad Nacional, Heredia, Costa Rica, correos electrónicos: luis.sandoval.murillo@una.cr, carlos.morera.beita@una.cr

** Escuela de Ciencias Biológicas, Universidad Nacional, Costa Rica, correo electrónico: ivan.sandoval.hernandez@una.cr
\end{abstract}


Key words: Geographical Information Systems (GIS), Crocodylus acutus, habitat, alteration, interaction.

\section{Resumen}

En los últimos años, la incidencia de ataques por cocodrilos en Costa Rica, se han incrementado, lo cual está asociado al desarrollo de las actividades humanas en áreas consideradas como óptimas en lo que determinó como hábitat potencial de la especie. Dado lo anterior, la presente investigación plantea la identificación de zonas de interacción entre el cocodrilo y el ser humano a partir de la integración de variables físico-geográficas por medio de los Sistemas de Información Geográfica (SIG). De esta manera, se realizó una recopilación teórica para la identificación de las variables físico-geográficas que integran el hábitat del cocodrilo (pendiente, red de drenaje, áreas de inundación y coberturas de uso de la tierra), así como las variables socioambientales que influyen en la alteración y la pérdida del hábitat de la especie (cantidad de poblados, densidad de población, densidad de caminos, y coberturas de uso de la tierra). Así mismo, se integraron los registros de casos con cocodrilos atendidos por el cuerpo de bomberos. Se logró evidenciar que la mayoría de incidentes se presentan en zonas con una alta interacción (áreas adonde se realizan una amplia variedad de actividades antrópicas), dentro del hábitat potencial de este reptil. Adicionalmente, los resultados obtenidos en la investigación constituyen un insumo importante en la toma de decisiones a nivel de política y ordenamiento territorial en la Región Pacífico Central, ya que en esta zona el turismo es una de las principales actividades económicas que puede llegar a ser amenazada por un ataque de ésta especie.

Palabras clave: Sistemas de Información Geográfica (SIG), Crocodylus acutus, hábitat, alteración, interacción.

\section{Resumo}

Nos últimos anos, a incidência de ataques por crocodilos na Costa Rica aumentou, e isso está associado ao desenvolvimento das atividades humanas em áreas consideradas como ótimas no que se definiu como hábitat potencial da espécie. Diante disso, a presente pesquisa propõe a identificação de zonas de interação entre o crocodilo e o ser humano a partir da integração de variáveis físico-geográficas por meio dos Sistemas de Informação Geográfica (SIG). Desta maneira, se realizou uma recompilação teórica para a identificação das variáveis físico-geográficas que integram o habitat do crocodilo (inclinações, rede de drenagem, áreas de inundação e coberturas de uso da terra), assim como as variáveis sócio-ambientais que influem na alteração e perda do habitat da espécie (quantidade e densidade de população, densidade de caminhos, e coberturas e uso da terra). Assim mesmo, se integraram os registros de casos com 
crocodilos atendidos pelo corpo de bombeiros. Evidencia-se que a maioria dos incidentes se apresentam em zonas com uma alta interação (áreas onde se realizam uma ampla variedade de atividades antrópicas), dentro do habitat potencial deste réptil. Adicionalmente, os resultados obtidos na pesquisa constituem um insumo importante na tomada de decisões a nível político e ordenamento territorial na Região do Pacífico Central, já que nesta zona o turismo é uma das principais atividades econômicas que pode chegar a ser ameaçado por um ataque desta espécie.

Palavras chave: Sistemas de Informação Geográfica (SIG), Crocodylus acutus, habitat, alteração, interação.

\section{Introducción}

El cocodrilo americano (Crocodylus acutus) se distribuye en ambas costas del continente americano, desde el sur de México hasta el norte de Suramérica. En Costa Rica, se reportan habitando ríos, lagos, pantanos, esteros, manglares, en elevaciones menores a los 700 msnm y en el mar en áreas cercanas a las costas (Bolaños et al., 1995). El Pacífico Central agrupa una de las mayores poblaciones de C. acutus, influenciado por una densa red de drenaje, la presencia de 16 cuencas hidrográficas de las 34 de mayor importancia que tiene el país, tales como: Bongo, Lagarto, Guacimal, Aranjuez, Barranca, Jesús María, Grande de Tárcoles, Tusubres, Parrita, Damas, Naranjo, Savegre y Barú.

Durante la década de los sesenta en Costa Rica, la intensa cacería generó que la especie fuera categorizada por la legislación nacional e internacional en peligro de extinción, medida que influyó en una progresiva recuperación de sus poblaciones. Éste incremento provocó la recolonización de sus antiguos hábitats, y acompañado de la ausencia de mapas de zonificación que impliquen restricciones o regulaciones para las actividades antrópicas, se generó un aumento de incidentes entre poblaciones humanas y cocodrilos, potencializados por factores como el cambio en la cobertura de uso de la tierra, vinculados a la invasión del hábitat natural de este reptil, así como al uso de las áreas de protección de ríos y quebradas por actividades agrícolas, situación que ha generado deterioro y reducción de sus principales fuentes de alimento, así como la disminución de los caudales de los ríos en la época seca o por la presencia de fenómenos climáticos que alteran su entorno natural, propiciando mayores interacciones entre humano-cocodrilo (García y Buenrostro, 2015).

A inicios de la década de los noventa, se identifica una mayor presencia de cocodrilos en sus zonas de distribución, así como un aumento de ataques hacia los seres humanos en los ríos Bebedero, Cañas, Tempisque y algunos ríos del Pacífico Central, estos incidentes se han asociado a la relación directa entre los seres humanos y el cocodrilo, producto probablemente de la invasión de su hábitat (Bolaños, 2012). En 
la actualidad, estas relaciones persisten debido al amplio desarrollo de actividades antrópicas asociadas a las vías de comunicación, crecimiento de la población, expansión residencial concentrada y dispersa, la cantidad de centros poblados por $\mathrm{km}^{2}$, así como las actividades agropecuarias (cultivos-pasto) en áreas costeras, en márgenes de ríos, esteros y cuerpos de agua. Dichas actividades han generado la alteración del medio natural afectando el hábitat de la especie y potencializando la interacción con el ser humano.

Considerando esta problemática, la presente investigación tuvo como objetivo identificar las áreas propensas a incidentes por ataques de cocodrilos (Crocodylus acutus) en el Pacífico Central de Costa Rica, para la identificación del nivel de amenaza, a partir de un Sistema de Información Geográfica. Donde se valoran aspectos físicos-geográficos, características del hábitat de la especie, así como las condiciones socio-ambientales relacionadas a la alteración de su entorno y el nivel de exposición de los seres humanos a la presencia de esta especie. Lo anterior, abordado desde la geografía, la cual como ciencia tiene como objeto de estudio el espacio y las relaciones sociedad-naturaleza, aportando en investigaciones concretas para la resolución de los problemas socio-ambientales, basado en técnicas como los Sistemas de Información Geográfica y los Sensores Remotos, por medio del modelaje de variables espaciales y su representación cartográfica.

\section{Área de estudio}

El área de estudio corresponde a la región Pacífico Central, creada mediante Decreto 16068-PLAN de febrero de 1985, que comprende los cantones de la provincia de Puntarenas: Esparza, Montes de Oro, Aguirre, Parrita, Garabito y Puntarenas, así como los de San Mateo y Orotina pertenecientes a la provincia de Alajuela. Se delimita por las coordenadas geográficas extremas $9^{\circ} 19^{\prime} 00^{\prime \prime}$ y $10^{\circ} 9$ ' 28" latitud norte; $83^{\circ} 57^{\prime} 36^{\prime \prime}$ y $85^{\circ} 06^{\prime} 20^{\prime}$, longitud oeste y las coordenadas métricas 382147 1123411 y 514551 - 1023484. Según la regionalización del SINAC la administración de la Región Pacífico Central, pertenece al Área de Conservación Pacífico Central (ACOPAC) (Mapa 1). En el presente estudio no se considera los distritos de Lepanto, Paquera e Isla Chira.

Las condiciones climáticas del Pacífico Central, de acuerdo con el Instituto Meteorológico Nacional (IMN, sf), se caracterizan por presentar el régimen de precipitación del Pacífico. Su posición geográfica al suroeste del país, con la protección al norte de cadenas montañosas impiden la incursión de vientos alisios del noreste, estableciéndose una modificación de este régimen del Pacífico, dando origen a la presencia de dos estaciones marcadas como el clima tropical seco, con una estación seca corta y moderada desde enero a marzo y un período lluvioso muy severo y largo dividido en dos períodos; el primero de mayo a agosto y el segundo de septiembre a octubre. Mientras que los meses de abril y diciembre están marcados como los meses 
de transición. La precipitación promedio de la zona es de $3500 \mathrm{~mm}$ anuales y la temperatura máxima promedio es de $31.0^{\circ} \mathrm{C}$ y la mínima promedio es de $22.7^{\circ} \mathrm{C}$.

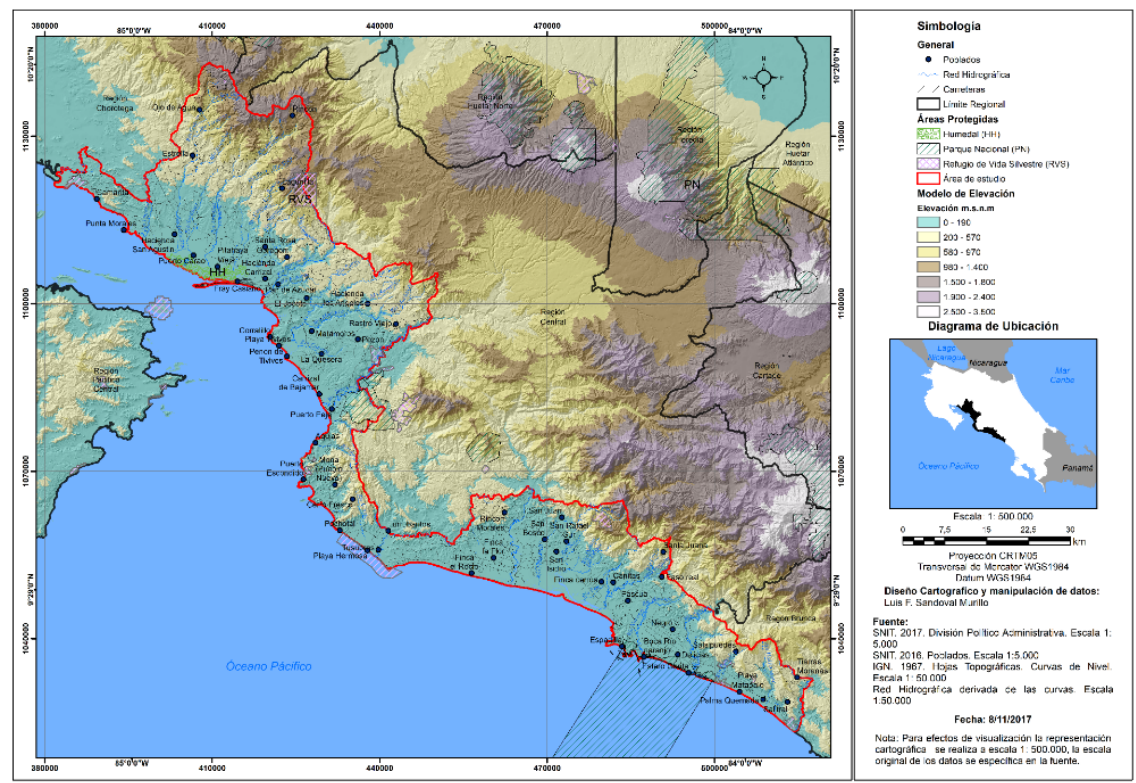

Mapa 1. Ubicación del área de estudios, Pacífico Central.

Se caracteriza por un sistema fluvial, perteneciente a la vertiente del Pacífico, donde sobresalen ríos como el Tárcoles, Jesús María, Guacimal y Aranjuez. Mismos que han configurado su geomorfología dando origen a extensas llanuras, en que se desarrollan actividades como la agricultura y la ganadería, sobresaliendo las llanuras del Tárcoles y Parrita (Astorga, sf).

\section{Metodología}

En esta investigación se identificaron dos escenarios, el primero referido al hábitat potencial del cocodrilo americano ( $C$. acutus), considerando elementos físico-geográficos como la altitud, red de drenaje, áreas de inundación y la delimitación de las áreas de manglar, ríos anchos y desembocaduras. El segundo escenario correspondiente a todas las actividades concernientes a la alteración del hábitat donde se consideraron elementos socio-ambientales como: densidad de las vías de comunicación, densidad de poblados, cantidad de población por distrito, coberturas de uso de la tierra (asentamientos, cultivos y pastos). A partir de los cuales se deriva la generación de las áreas de interacción entre el ser humano y este reptil, proceso que se describe a continuación: 
En la realización del primer escenario se elaboró el Modelo Digital de Elevaciones (MDE) a partir de la capa vectorial de curvas a escala 1:50 000. Posteriormente, se realizó un proceso de interpolación para la obtención del MDE con la herramienta de interpolation / topo to raster del ArcGis, el cual es definido por ESRI como un método de interpolación diseñado específicamente para crear modelos digitales de elevación (DEM) hidrológicamente correctos, el cual está basado en el programa ANUDEM, desarrollado por Hutchinson en el periodo 1988-2011. Una vez realizado el MDE se deriva la red de drenaje, con la aplicación de Hidrology del ArcGIS, la cual se usa en la delimitación de cuencas hidrográficas, con el fin de que los cauces de los ríos y quebradas coincidan con el MDE y evitar el desplazamiento de las capas de ríos existentes. Con la generación de la red hídrica en el área de estudio se utilizó la aplicación de density/Kernel Density, para estimar la densidad de ríos por $\mathrm{km}^{2}$, con el fin de facilitar la integración de las variables posteriormente, cada una es clasificada en tres categorías (alta, media y baja) utilizando la clasificación igualitaria por intervalo.

Se consideraron las áreas de inundación correspondientes a la demarcación oficial realizada por la Comisión Nacional de Emergencias, las cuales se ajustaron al MDT para evitar desplazamiento. A las áreas que no presentaban riesgo de inundación se asignaron valores de cero. Otra de las variables identificadas a partir de la literatura existente para el hábitat del cocodrilo son las coberturas de manglares, cauces de ríos, desembocaduras y cuerpos de agua, que fueron identificadas utilizando imágenes RapidEye a escala 1:25000, mediante la técnica de fotointerpretación y las herramientas de edición del programa ArcGis 10.5. Una vez identificadas y procesadas las capas en formato vectorial se procede a su integración para la obtención del hábitat potencial del cocodrilo.

Para el segundo escenario, relacionado a la alteración del hábitat por actividades humanas se procesaron las capas vectoriales: red de caminos para generar un ráster de densidad de caminos en $\mathrm{km}^{2}$, con base a la metodología implementada por Ramírez, Jiménez y Martínez (2005), la cual consiste en la utilización de un Sistema de Información Geográfica (SIG) para realizar el análisis de la estructura y de la densidad de la red caminos. Esta última definida como el promedio de la longitud total de caminos por unidad de área $\left(\mathrm{km} / \mathrm{km}^{2}\right)$. Específicamente, se utilizó la extensión Density Kernel, procesamiento aplicado a la capa vectorial de poblados con el fin de generar áreas continúas para su posterior análisis.

La identificación de las coberturas de uso de la tierra que muestran el desarrollo de actividades humanas se elaboró utilizando las imágenes satelitales LandSat con una resolución espacial de 30 metros para los años 2000 y 2015. Adicionalmente, se aplicó una corrección radiométrica y atmosférica, la cual, según Ambrosio et al. (sf), es necesaria para modelar adecuadamente parámetros físicos realistas y consistentes, 
al abordar estudios multi-temporales, mismos que facilitan el proceso de clasificación supervisada y de fotointerpretación. Las categorías de uso identificadas se definen en la Tabla 1.

\section{Tabla 1}

Definición de las categorías de uso de la tierra

\begin{tabular}{|c|c|}
\hline Categoria & Descripción \\
\hline Bosque en pendiente alta & $\begin{array}{l}\text { Cobertura vegetal dominada por árboles de copas anchas y } \\
\text { casi continuas, en pendientes mayores a } 30 \%\end{array}$ \\
\hline Bosque en pendiente baja & $\begin{array}{l}\text { Cobertura vegetal con la presencia de elementos arbóreos y } \\
\text { arbustivos con copas menos continuos. Han sido interveni- } \\
\text { das y se encuentran en regeneración, en pendientes menores } \\
\text { a } 30 \%\end{array}$ \\
\hline Pasto en pendiente alta & $\begin{array}{l}\text { Supresión total de los bosques por perturbaciones humanas } \\
\text { y substitución por gramínea para ganadería intensiva en pen- } \\
\text { dientes mayores al } 30 \%\end{array}$ \\
\hline Pasto en pendiente baja & $\begin{array}{l}\text { Supresión total de los bosques por perturbaciones humanas } \\
\text { y substitución por gramínea para ganadería intensiva en pen- } \\
\text { dientes menores al } 30 \%\end{array}$ \\
\hline Manglar & $\begin{array}{l}\text { Bosques pantanosos que ocupan espacios con alta sedimen- } \\
\text { tación donde se mezcla el ambiente marino y el terrestre }\end{array}$ \\
\hline Cuerpos de agua & $\begin{array}{l}\text { Áreas de lagos, lagunas y del curso de agua (río principal y } \\
\text { tributarios) }\end{array}$ \\
\hline Cultivos & $\begin{array}{l}\text { Son áreas con ocupación agrícola que puede ser permanentes } \\
\text { o estaciónales }\end{array}$ \\
\hline Asentamientos humanos & $\begin{array}{l}\text { Están representados por la ocupación urbano-residencial dis- } \\
\text { persa, acompañado de servicios públicos institucionales, in- } \\
\text { dustrial, comercial }\end{array}$ \\
\hline
\end{tabular}

Fuente: $\quad$ adaptado de Morera y Sandoval, 2015.

Con la realización de cada una de las variables del primer y segundo escenario, se realizó las clasificación de cada una de las capas, donde cada uno de los valores se normalizaron, lo cual consiste en la estandarización de los pesos asignados mediante el método de máximo de lista, que se fundamenta en dividir el peso de cada registro asignado entre el valor máximo de los pesos por cada una de las categorías, obteniendo así un valor normalizado para cada registro, que oscila en un rango de 0 a 1 (Submitted, Fulfillment, y Kong, 2010). Siendo 1 el valor óptimo y 0 el menos óptimo para cada una de las variables a relacionar.

$$
x_{i}^{\prime}=x_{i} / x_{\max }
$$


Una vez realizada la normalización se establecieron tres grupos de categorías por valor, según los pesos normalizados asignados a cada variable y clasificados en tres rangos: alta, moderada y baja. Una vez determinados los rangos se relacionan por medio de un álgebra de mapas (suma), utilizando la media o promedio correspondiente a la suma de todas las puntuaciones de la variable dividida por el número total de casos, Fierro (2010). Obteniendo dos modelos espaciales, los cuales son representados cartográficamente, relacionados al hábitat potencial (topografía, red de drenaje y coberturas de uso de la tierra) y el mapa de la alteración del hábitat (la densidad de caminos, densidad de población y la distancia a centros poblados). Con la generación de ambos modelos, se procede a establecer la relación entre ambos para determinar cuáles son los sectores que representan mayor interacción entre el ser humano y el cocodrilo, utilizando el mismo método descrito anteriormente, por lo que se constituye la siguiente clasificación para los mapas de hábitat potencial y alteración del hábitat, Tabla 2.

Tabla 2

Normalización del hábitat potencial y alteración del hábitat

\begin{tabular}{ccc}
\hline \multicolumn{3}{c}{ Hábitat potencial } \\
\hline Clasificación & Peso & Normalización \\
\hline Alta & 3 & 1 \\
Moderada & 2 & 0.66 \\
Baja & 1 & 0.33 \\
\hline \multicolumn{3}{c}{ Alteración del hábitat } \\
\hline Clasificación & Peso & 1 \\
Alta & 3 & 0.66 \\
Moderada & 2 & 0.33 \\
Baja & 1 &
\end{tabular}

Fuente: elaboración propia.

Con la generación de los dos escenarios descritos, se desarrolló el mapa de interacciones entre el ser humano y el cocodrilo, donde se realizó el mismo procedimiento descrito en el hábitat potencial y alteración del hábitat, donde la integración de los valores se agrupó en tres categorías como se muestra en la Tabla 3.

Con la realización de las zonas de interacción entre ambas especies se evidencian los incidentes con cocodrilos, atendidos y registrados por el cuerpo de bomberos para el periodo 2007-2017, los cuales eran los únicos registros que contenían una localización espacial. 
Tabla 3

Zonas de interacción entre el cocodrilo y el ser humano

\begin{tabular}{ccc}
\hline ID & Rango de la sumatoria & Categorías \\
\hline 1 & $0.33-0.495$ & Baja \\
2 & $0.4951-0.665$ & Media \\
3 & $0.6651-1$ & Alta \\
\hline
\end{tabular}

Fuente: elaboración propia.

\section{Resultados y discusión}

\section{Dinámicas ecológicas y hábitat del cocodrilo}

La distribución de los cocodrilos se encuentra influenciada por elementos como la altitud, la hidrografía, el tipo de cobertura y la presencia de áreas de inundación, lo cual, de acuerdo con Bolaños, Sánchez y Piedra (1995), son condiciones que delimitan su hábitat a sectores específicos, abarcando principalmente áreas de manglar, ríos anchos y caudalosos. Todas estas prevalecen en el Pacífico Central en ríos como: Jesús María, Tivives y El Grande de Tárcoles. Adicionalmente, la distribución y densidad de los individuos puede afectarse por los patrones de migración o dispersión que estos efectúan, principalmente durante la época de apareamientos, comprendida, en general, de agosto a diciembre, meses que se catalogan como los más peligrosos, ya que los machos dominantes desplazan a los machos subordinados a hábitats de baja calidad ( sitios sin hembras, con baja cobertura boscosa, con cercanía a poblados, zonas de ríos angostos y contaminados), llegando estos animales a áreas donde normalmente no se encuentran fuera de la época reproductiva. Esta situación, potencia la interacción entre los seres humanos y la especie (Sandoval, 2016).

Por otra parte, durante la época lluviosa, comprendida entre mayo a noviembre, los cocodrilos tienden a distribuirse aleatoriamente por cuerpos de agua que se forman durante fuertes precipitaciones y, posteriormente durante la época seca tienden a localizarse en los cauces principales de ríos o lagunas que mantienen agua aún durante la ausencia de precipitación. De la misma manera, cuando el río baja su nivel y/o se extrae gran cantidad de agua, muchos cocodrilos encuentran sus espacios en canales de riego o estanques de las empresas que se dedican a actividades de cultivo de peces y actividades agrícolas (Sánchez, 2001a). Con respecto a su densidad, en Costa Rica se han en encontrado abundancias relativas por $\mathrm{km}^{2}$ de nueve cocodrilos en los ríos Tempisque, cinco en el Tárcoles y cinco en el Térraba-Sierpe, asimismo se han observado en sitios donde normalmente no se les encontraba. Sin embargo, no existe un censo de la cantidad de individuos por hábitat, por lo cual es erróneo referirse a una sobrepoblación de la especie (Estado de la Nación, 2008). 
Sin embargo, la invasión del hábitat del cocodrilo perturba el comportamiento normal de la especie, porque a pesar de ser animales muy resistentes, son vulnerables a los constantes cambios de la cobertura de uso de la tierra, producto de las actividades antrópicas (mismas que invaden su hábitat, incidiendo en la destrucción de la vegetación, alteración y modificación de los márgenes de los ríos), afectando los sitios de postura, asoleo, y distribución en general de la especie. Además, producto de la interacción con humanos se destruyen las cuevas que utilizan los animales para ocultarse del peligro o, eventualmente descansar o guardar alimento. Es muy importante señalar la afectación del comportamiento propio de la especie, producto de actividades turísticas en áreas de su distribución. De esta manera, la dinámica mencionada incide en la variación de las características y condiciones de su hábitat, los animales están obligados a migrar a otros sitios donde las condiciones naturales les ofrezcan protección y alternativas para sus actividades de descanso, asoleo, alimentación, protección y anidamiento (Sánchez, 2001b).

Sandoval y Bolaños (2016) argumentan que la fragmentación del paisaje en los últimos años en la región Pacífico Central y el Gran Humedal Tempisque está relacionada con una mayor interacción entre el cocodrilo y el ser humano, características que están determinadas por la distribución espacial, la forma, el tamaño del fragmento y el grado de conectividad entre las unidades de paisaje, acciones que generarían consecuencias como pérdida y reducción del hábitat de la especie. Además, ésta fragmentación incrementa la presencia de especies generalistas como ratas (Rattus), pizotes (Nasua Narica), mapaches (Procyon lotor), zorros pelones (Didelphis virginiana), así como espacios con alta densidad humana con frecuencia de animales domésticos como perros, gatos, gallinas, cerdos, entre otros que pueden convertirse en presa fácil para los cocodrilos (Cortijo, sf).

Con respecto al hábitat potencial del cocodrilo, son diversos los factores que se relacionan para la determinación del mismo como: la altitud menor a los $700 \mathrm{msnm}$, la presencia de áreas de manglar, acompañada de ríos caudalosos y anchos, la existencia de playones, así como las áreas de inundación mismas que funcionan de medio de desplazamiento durante la crecida de los ríos en presencia de fuertes precipitaciones o fenómenos atmosféricos prolongados, todos son un conjunto de variables que posibilitan la demarcación de su hábitat espacial dentro de un territorio, para el caso de la Región Pacífico Central se estableció la presencia de áreas propensas a la presencia del cocodrilo, como se refleja en el Mapa 2 y la Tabla 4, donde se muestra la distribución espacial del hábitat potencial en tres sectores contemplados como; alto, medio y bajo.

El hábitat potencial alto se caracteriza por elementos como cuerpos de agua, una alta densidad de la red de drenaje (1 $862-3053 \mathrm{~km}^{2}$ ), donde se destacan ríos importantes como el Jesús María, Tivives y El Grande de Tárcoles, además de manglares, esteros, 
elevaciones menores a los $240 \mathrm{msnm}$, y áreas propensas a inundaciones, representando el $21.5 \%$ del Pacífico Central. Estas condiciones posibilitan la presencia del cocodrilo, debido a que pueden reunir las variables óptimas características de su hábitat, que de acuerdo con Bolaños, Sánchez y Piedra (1995) son condiciones que delimitan su hábitat a sectores específicos, abarcando principalmente áreas de manglar, ríos anchos y caudalosos. Todas estas condiciones que prevalecen en ríos como el Grande de Tárcoles y en el Jesús María.

Tabla 4

Región Pacífico Central, Clasificación del hábitat potencial y superficie

\begin{tabular}{lcc}
\hline Clasificación hábitat & Hectáreas & Porcentaje \\
\hline Hábitat Potencial Alto & 59484.0 & 21.5 \\
Hábitat Potencial Bajo & 117781.4 & 42.5 \\
Hábitat Potencial Medio & 99658.0 & 36.0 \\
Total & 276923.4 & 100 \\
\hline
\end{tabular}

Fuente: elaboración propia.

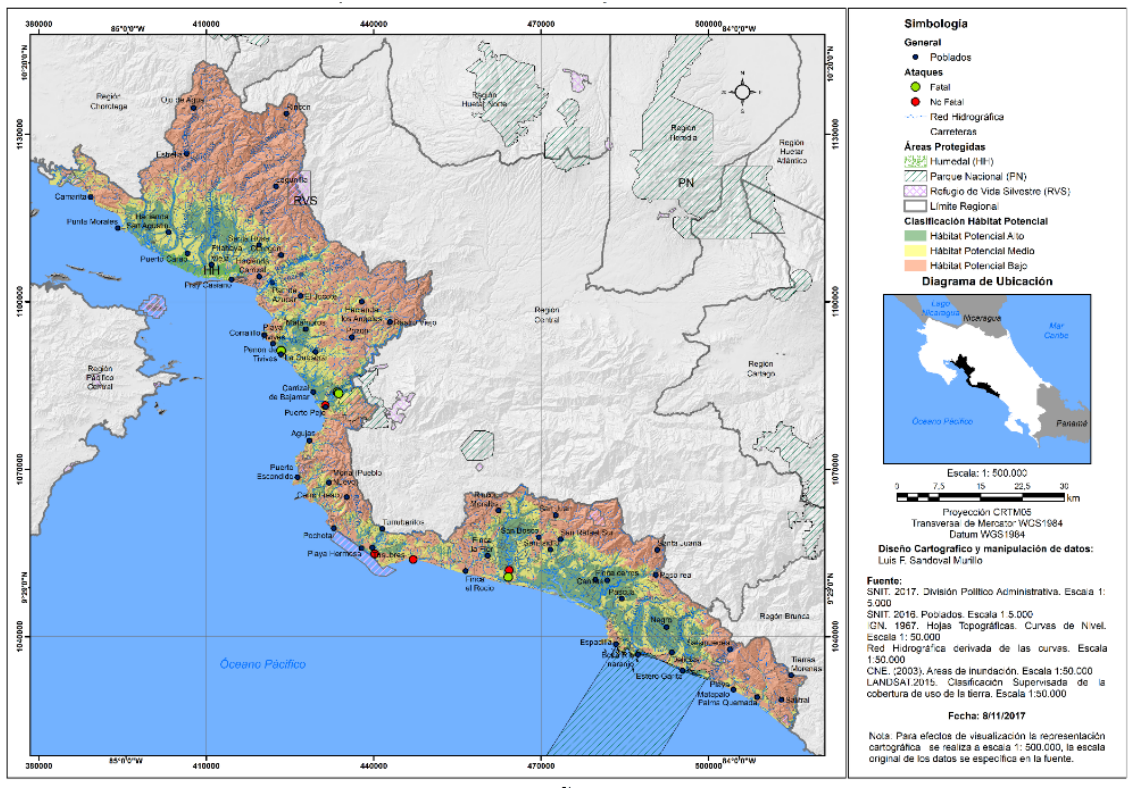

Mapa 2. Hábitat potencial del Crocodylus acutus, Pacífico Central Costa Rica. 
Con respecto al hábitat potencial medio, se presenta una moderada densidad de ríos por km² entre 1 196-1 $861 \mathrm{~km}^{2}$, donde se da la presencia de coberturas de uso de la tierra como pasto y bosque en pendientes moderadas, cuerpos de agua, cultivos y elevaciones entre 234-466 msnm, representando un 42.5\% de la Región (Mapa 2). Estas condiciones son poco viables para la presencia del cocodrilo, ya que no presentan las características necesarias para su hábitat. Con respecto al hábitat potencial, las posibilidades de la presencia del cocodrilo van de escasas a nulas, debido a que las condiciones de esta categoría muestran una insuficiente densidad de la red de drenaje por $\mathrm{km}^{2}(0.074-1.195)$, nula presencia de cuerpos de agua y manglares, y pendientes superiores a los $467 \mathrm{msnm}$. Abarcando una superficie de un 36\% de la región.

\section{Alteración del hábitat potencial del cocodrilo}

La integración de las variables antrópicas que constituyen la alteración del medio, producto de su desarrollo sobre el espacio geográfico, se vinculan a el aumento en la infraestructura y actividades como: vías de comunicación (densidad por $\mathrm{km}^{2}$ ), crecimiento de la población por distrito, asociado a la expansión residencial concentrada y dispersa, actividades agropecuarias y la cantidad de centros poblados por $\mathrm{km}^{2}$ en el PC. Condiciones que se reflejan en investigaciones realizadas por Bolaños (2011), donde se evidencia la invasión y alteración del hábitat del cocodrilo en las últimas décadas, producto del crecimiento demográfico, el avance de la frontera agrícola, la presión urbana, la construcción y mejoramiento de la red vial, consecuentemente, con la generación de la fragmentación de los ecosistemas. Su combinación espacial permite visualizar la agrupación de actividades en el territorio y relacionarlo con el grado de alteración del hábitat potencial del cocodrilo en el Pacífico Central.

La Tabla 5, muestra las áreas de alteración por el desarrollo de actividades humanas en la región, donde un 20.4\% presenta alta alteración de las condiciones naturales, producto del dinamismo de cultivos, áreas residenciales dispersas, distritos con una alta cantidad de habitantes como el Roble, Chacharita, Jaco y Parrita, mismos que reúnen una alta densidad de vías por $\mathrm{km}^{2}$, entre 1.56-2.33, así como una alta densidad de poblados. Con respecto al hábitat potencial delimitado anteriormente, el porcentaje de alteración corresponde a un $8.47 \%$, evidenciando una alta degradación, lo que incrementa la interacción entre el cocodrilo y el ser humano (Mapa 3), producto del rápido crecimiento y establecimiento de asentamientos humanos en las últimas décadas, atribuido a políticas de desarrollo agropecuario que propiciaron la demanda de tierras, ocupando las llanuras de inundación, esteros y lagunas costeras, las cuales son habitadas por el Crocodylus acutus (García y Buenrostro, 2015). 
Tabla 5

Alteración del hábitat potencial del cocodrilo americano

\begin{tabular}{ccc}
\hline \multicolumn{3}{c}{ Alteración hábitat región } \\
\hline Clasificación & hectáreas & porcentaje \\
\hline Alta alteración del hábitat & 55913.96 & 20.44 \\
Baja Alteración del hábitat & 110718.42 & 40.47 \\
Moderada alteración del hábitat & 106973.39 & 39.10 \\
Total & 273605.77 & 100 \\
\hline Clasificación & hectáreas & Porcentaje \\
\hline Alta alteración del hábitat & 23187.71 & 8.47 \\
Moderada alteración del hábitat & 22619.32 & 8.27 \\
Baja alteración del hábitat & 12206.53 & 4.46 \\
Total & 58013.56 & 100 \\
\hline
\end{tabular}

Fuente: elaboración propia.

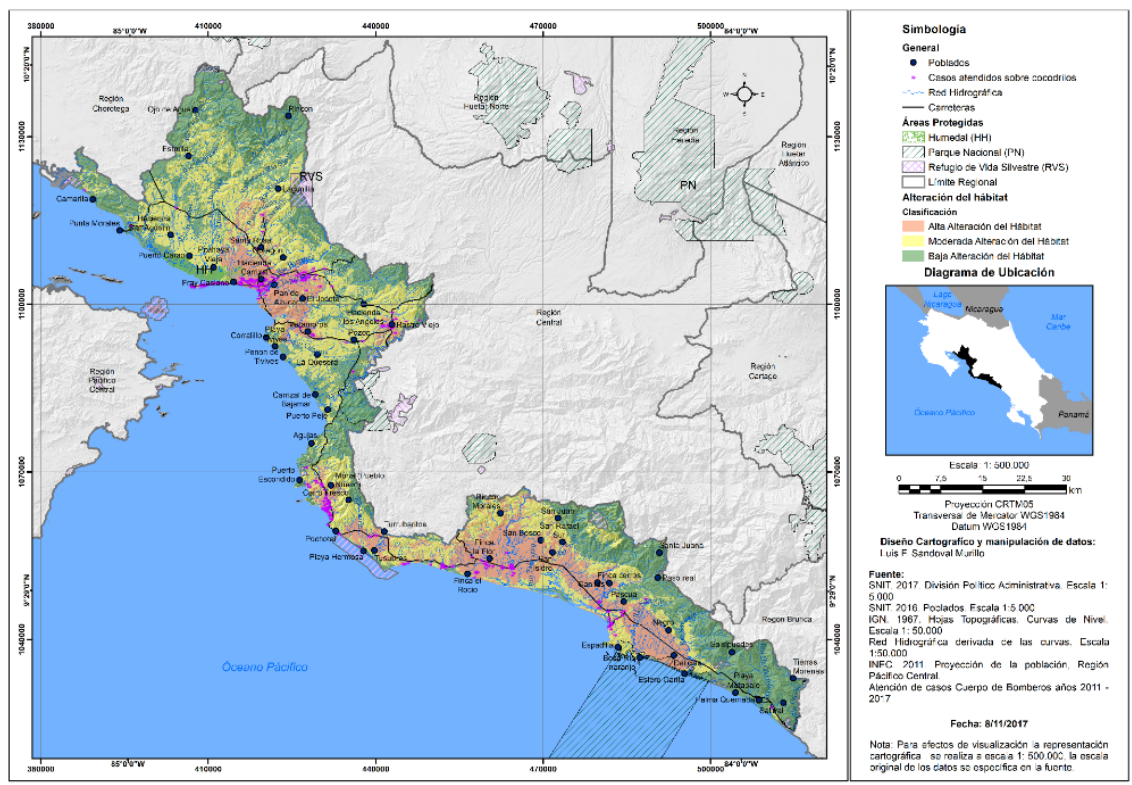

Mapa 3. Alteración del hábitat potencial del cocodrilo, Pacífico Central Costa Rica. 
La superficie ocupada por una moderada alteración del hábitat corresponde a un $40 \%$ para la totalidad de la región, considerando únicamente la alteración del hábitat potencial alto de la especie, representa un $8.27 \%$, producto de la presencia de actividades humanas de forma no intensificada, pero que de igual forma influye en la alteración del entorno natural, conllevando a la interacción directa con la vida silvestre. Con respecto a las áreas de baja alteración, estas corresponden a sectores con una fuerte irregularidad del terreno, que imposibilitan la presencia de actividades humanas, las que representan un $40.47 \%$ del Pacífico Central y un $4.46 \%$ del hábitat potencial de la especie, en el primer caso, por sus características geomorfológicas imposibilitan la presencia del cocodrilo.

\section{Zonas de interacción entre el ser humano y el cocodrilo}

La realización de este apartado se fundamentó en la modelización del hábitat potencial abordado anteriormente, considerando las condiciones óptimas para la presencia de C. acutus, a partir de la relación de las variables que caracterizan su hábitat, como elevaciones menores a los $700 \mathrm{msnm}$, una alta densidad de la red hidrográfica, la presencia de manglar, playones de río y áreas propensas a inundaciones (Bolaños, 2002). En este aspecto es significativo resaltar lo establecido por Santillán (2013), cuando se realiza la modelación de la distribución de una especie, cuando afirma que la presencia de todas las variables ambientales óptimas en un determinado hábitat no garantiza la existencia efectiva de la especie. Asociando la dispersión a factores reproductivos de la especie, así como las alteraciones generadas por las actividades humanas que inciden en la fragmentación ecosistémica y constituye barreras geográficas.

De acuerdo con Sandoval, Morera y Sandoval (en prensa), el hábitat potencial del cocodrilo en el Pacífico Central durante el periodo 2000-2015 ha experimentado cambios de uso y la fragmentación de su paisaje en coberturas como el manglar, cuerpos de agua y bosque, presentándose un incremento en el número de fragmentos asociados a una reducción en su superficie. Lo anterior es resultado de la alteración y la presión a la que han sido sometidas estas coberturas en áreas consideradas como óptimas para la presencia del cocodrilo, producto del desarrollo de actividades antrópicas. Dinámicas que se evidencian en los últimos años a partir de reportes de periódicos, atención de casos por Bomberos, reportes al servicio 911 y registros realizados por asociaciones como CrocBITE, así como recopilaciones de casos por investigadores como Bolaños (2017).

A partir de la combinación del hábitat potencial y las áreas de interacción por actividades humanas, considerando únicamente los sectores determinados como hábitat potencial alto para el cocodrilo, se obtuvo la representación espacial de las zonas que presentan la posibilidad de una mayor interacción entre el ser humano y el cocodrilo, misma que podría potenciar el encuentro entre ambas especies, que tal como lo 
menciona Andrade (2013) se han incrementado en los últimos 40 años, potencializado por las actividades antrópicas en áreas naturales donde habita el cocodrilo.

La Figura 1, muestra las áreas de alta interacción entre el ser humano y el cocodrilo en el Pacífico Central con un $20.44 \%$ de su superficie, de la cual un $6.5 \%$, se localiza dentro del hábitat potencial alto de la especie (Mapa 4), donde se desarrollan actividades antrópicas con una alta concentración como densidad de carreteras por $\mathrm{km}^{2}$, cantidad de población, densidad de poblados por $\mathrm{km}^{2}$ y amplio desarrollo de actividades agrícolas y turísticas. De acuerdo con Bolaños (2002), la alteración del hábitat del cocodrilo se ve afectada por el incremento y desarrollo de vías de comunicación, las cuales influyeron en el acrecentamiento del tránsito de personas en diferentes lugares, y un lógico aumento de la población humana, derivando en el surgimiento y expansión de asentamientos antrópicos, en detrimento de las áreas silvestres, incidiendo en la fragmentación de los ecosistemas. Por otra parte, el amplio desarrollo antrópico que se refleja en los datos expuestos anteriormente y la atención de casos por parte del cuerpo de bomberos entre el periodo 2007-2017, colaboran con lo obtenido en la zonificación de las áreas de interacción entre ambas especies. Además, coinciden con los sitios donde se han registrado más accidentes por cocodrilos, relacionados con el río Parrita, y en áreas cercanas al mar, sobresaliendo ataques en playa Esterillos, ambas áreas que concuerdan con las zonas de alta interacción.

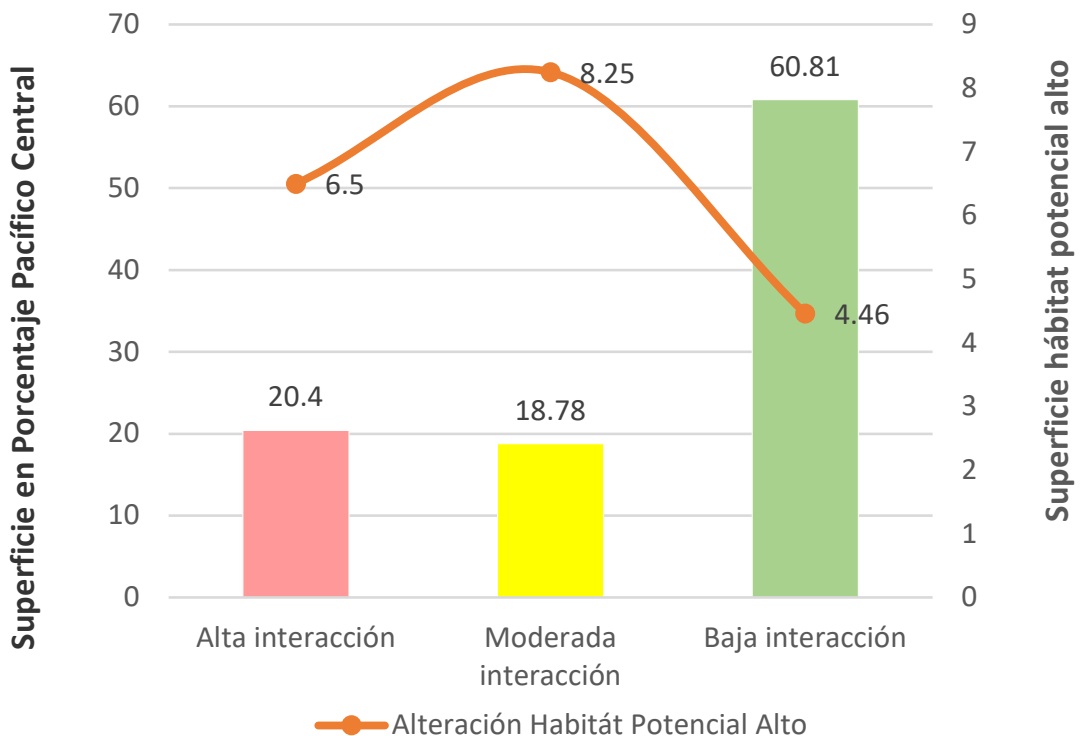

Figura 1. Pacífico Central: zonas de interacción entre el ser humano y Crocodylus acutus. 
Las zonas de moderada interacción abarcan un 18.78\% del Pacífico Central, sin embargo, dentro de las condiciones moderadas para la presencia del cocodrilo se representa un $8.25 \%$, en distritos como: Savegre, Quepos, Parrita, Tárcoles, Pithaya, Chomes y Manzanillo (Mapa 4). Con respecto a las zonas de baja interacción, esta corresponde a sectores donde aún se conservan las condiciones naturales como manglares y la presencia de las actividades antrópicas son escasas o nulas correspondiendo a un $60.81 \%$ en la totalidad del área de estudio y constituye un $4.46 \%$ en el hábitat potencial de la especie. La Figura 2 muestra la cantidad de casos atendidos en las zonas de interacción entre ambas especies, donde la mayor cantidad de casos se registran en zonas con una alta interacción entre el periodo 2011-2017, destacando el año 2012 con la mayor cantidad de atenciones con un total de 114, el 2014 registra la atención de 22 casos y los años 2015 y 2016 un total de 28, con respecto al 2017 la disponibilidad de los datos son registrados al mes de julio, por lo que se muestra una disminución de los casos atendidos (16). Lo anterior, según lo expresado por Magaña et al. (2010), quienes indican que la ocurrencia de ataques de cocodrilos, sin provocación de los humanos, responden a la defensa territorial, defensa de nidos y/o crías, caza para alimentación, confusión de identidad o humano como objetivo secundario y autodefensa.

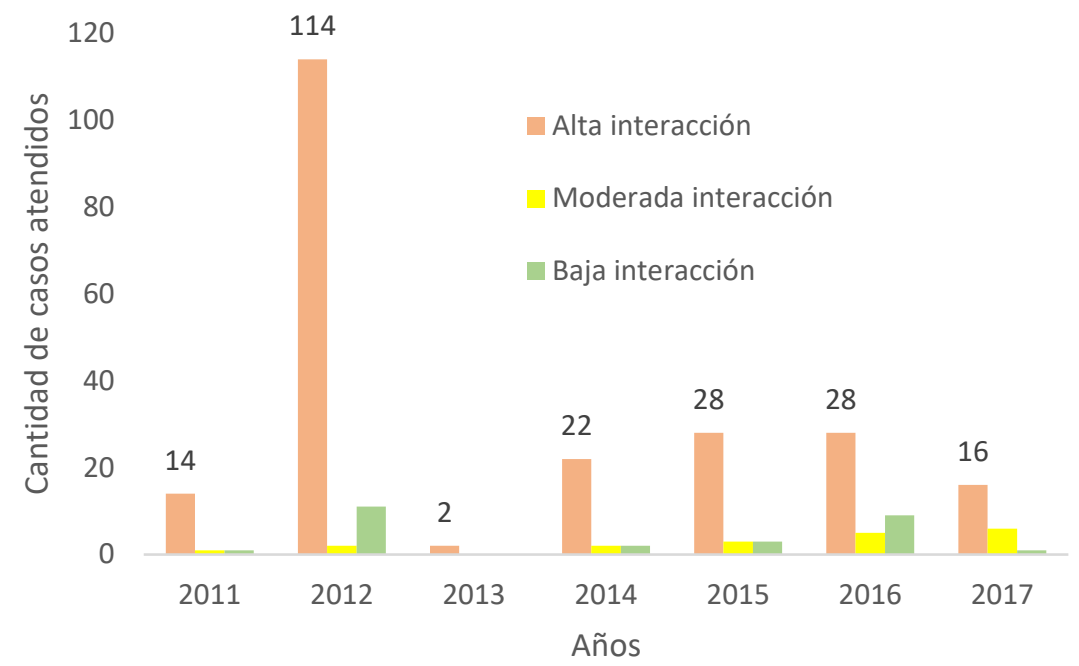

Figura 2. Pacífico Central: cantidad de casos atendidos según zona de interacción.

En la Tabla 6 se muestran los poblados que se localizan en las zonas de alta interacción catalogados como hábitat potencial del cocodrilo, los cuales se ubican dentro de los distritos de Quepos (Delicias, Damas, San Rafael y otros), Parrita (Pueblo 
Nuevo, San Isidro, Playa Sur y Julieta), Jaco (Turrubaritos), Ceiba (Machuca y Matamoros), Barranca (Sitio Junta) y San Juan Grande (Jesús María). La presencia de esta diversidad de poblados en sectores donde se reúnen las condiciones óptimas para la presencia de la especie constituye una zona de alto potencial de interacción entre el ser humano y el cocodrilo, el cual podría aumentar la atención de casos y efectos negativos hacia la población antrópica.

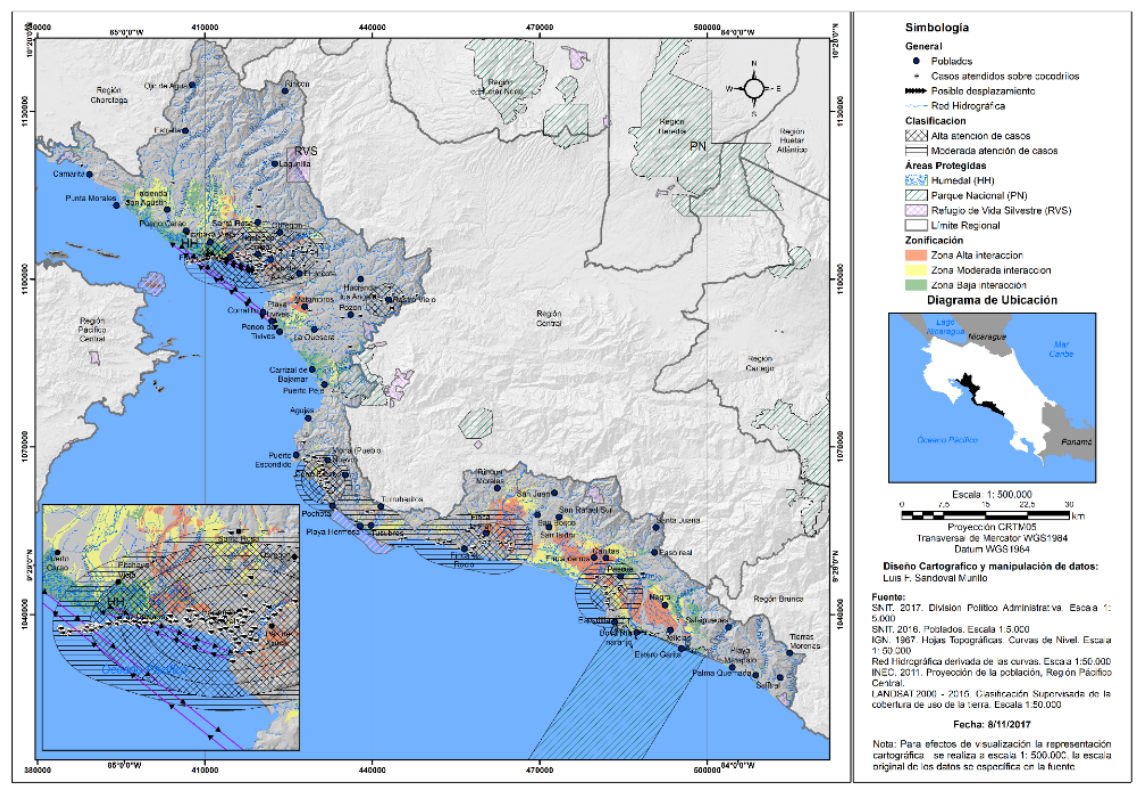

Mapa 4. Zonas de interacción entre el ser humano y el Crocodylus acutus, Pacífico Central Costa Rica.

Tabla 6

Poblados ubicados en las zonas de alta interacción en el hábitat potencial del Crocodylus acutus

\begin{tabular}{|c|c|c|c|c|c|}
\hline $\begin{array}{l}\text { Tipo de } \\
\text { poblado }\end{array}$ & $\begin{array}{l}\text { Nombre } \\
\text { Poblado }\end{array}$ & Distrito & $\begin{array}{l}\text { Tipo de } \\
\text { poblado }\end{array}$ & $\begin{array}{l}\text { Nombre } \\
\text { Poblado }\end{array}$ & Distrito \\
\hline Poblado & Delicias & Quepos & $\begin{array}{l}\text { Cabecera de } \\
\text { cantón }\end{array}$ & Parrita & Parrita \\
\hline Poblado & Mona & Quepos & Barrio & $\begin{array}{l}\text { Pueblo } \\
\text { Nuevo }\end{array}$ & Parrita \\
\hline $\begin{array}{l}\text { Hacienda/ } \\
\text { finca }\end{array}$ & $\begin{array}{l}\text { Finca } \\
\text { Pastora }\end{array}$ & Quepos & Poblado & Cabas & Quepos \\
\hline $\begin{array}{l}\text { Hacienda/ } \\
\text { finca }\end{array}$ & $\begin{array}{l}\text { Finca } \\
\text { Llorona }\end{array}$ & Quepos & Poblado & $\begin{array}{l}\text { Pirras (Las } \\
\text { Parcelas) }\end{array}$ & Parrita \\
\hline Poblado & Llorona & Quepos & Barrio & Julieta & Parrita \\
\hline
\end{tabular}


Continuación Tabla 6

\begin{tabular}{|c|c|c|c|c|c|}
\hline Poblado & Roncador & Quepos & Poblado & Pocares & Parrita \\
\hline $\begin{array}{l}\text { Hacienda/ } \\
\text { finca }\end{array}$ & $\begin{array}{l}\text { Finca } \\
\text { Roncador }\end{array}$ & Quepos & Poblado & Tigre & Parrita \\
\hline Poblado & Bartolo & Quepos & Poblado & $\begin{array}{l}\text { San } \\
\text { Rafael Norte }\end{array}$ & Parrita \\
\hline Poblado & Llamaron & Quepos & Poblado & Jicote & Parrita \\
\hline $\begin{array}{l}\text { Hacienda/ } \\
\text { finca }\end{array}$ & $\begin{array}{l}\text { Finca } \\
\text { Bartolo }\end{array}$ & Quepos & Poblado & Teca & Parrita \\
\hline Poblado & Managua & Quepos & Poblado & Sardinal Sur & Parrita \\
\hline Poblado & $\begin{array}{l}\text { Junta } \\
\text { Naranjo }\end{array}$ & Quepos & Poblado & $\begin{array}{l}\text { Quebrada } \\
\text { Amarilla }\end{array}$ & Jacó \\
\hline Poblado & Paquita & Quepos & Poblado & San Isidro & Parrita \\
\hline Poblado & Paquita & Quepos & Poblado & Turrubaritos & Jacó \\
\hline $\begin{array}{l}\text { Hacienda/ } \\
\text { finca }\end{array}$ & Finca Anita & Quepos & Poblado & Playa Sur & Parrita \\
\hline Poblado & $\begin{array}{l}\text { Estero } \\
\text { Damas }\end{array}$ & Quepos & Poblado & I Griega & Parrita \\
\hline Poblado & Anita & Quepos & Poblado & Surubres & Parrita \\
\hline Poblado & Ríos & Quepos & $\begin{array}{l}\text { Hacienda/ } \\
\text { finca }\end{array}$ & $\begin{array}{l}\text { Finca Brazo } \\
\text { Seco }\end{array}$ & $\begin{array}{l}\text { San Juan } \\
\text { grande }\end{array}$ \\
\hline Poblado & Papaturro & Quepos & Sitio & Sitio Uvita & Ceiba \\
\hline Poblado & Damas & Quepos & Poblado & Matamoros & Ceiba \\
\hline Poblado & Damas & Quepos & Sitio & Sitio Tablas & Ceiba \\
\hline Poblado & Cabitas & Quepos & $\begin{array}{l}\text { Hacienda/ } \\
\text { finca }\end{array}$ & $\begin{array}{l}\text { Hacienda } \\
\text { Mata de } \\
\text { Guinea }\end{array}$ & $\begin{array}{l}\text { San Juan } \\
\text { grande }\end{array}$ \\
\hline Poblado & Cerritos & Quepos & $\begin{array}{l}\text { Hacienda/ } \\
\text { finca }\end{array}$ & $\begin{array}{l}\text { Finca } \\
\text { Trinidad } \\
\text { Nueva }\end{array}$ & Ceiba \\
\hline $\begin{array}{l}\text { Hacienda/ } \\
\text { finca }\end{array}$ & $\begin{array}{l}\text { Finca } \\
\text { Cerros }\end{array}$ & Quepos & Poblado & Machuca & Ceiba \\
\hline Poblado & Cerros & Quepos & Poblado & Jesús María & $\begin{array}{l}\text { San Juan } \\
\text { grande }\end{array}$ \\
\hline Poblado & Cerros & Quepos & Sitio & $\begin{array}{l}\text { Sitio } \\
\text { Palmichal }\end{array}$ & $\begin{array}{l}\text { Espíritu } \\
\text { Santo }\end{array}$ \\
\hline Poblado & Gallega & Quepos & Poblado & Gregg & $\begin{array}{l}\text { Espíritu } \\
\text { Santo }\end{array}$ \\
\hline $\begin{array}{l}\text { Hacienda/ } \\
\text { finca }\end{array}$ & Finca Pirris & Quepos & Sitio & Sitio Junta & Barranca \\
\hline Poblado & San Rafael & Quepos & $\begin{array}{l}\text { Hacienda/ } \\
\text { finca }\end{array}$ & $\begin{array}{l}\text { Finca San } \\
\text { Manuel }\end{array}$ & Pitahaya \\
\hline Poblado & San Rafael & Quepos & Poblado & Barbudal & Miramar \\
\hline
\end{tabular}

Fuente: elaborado a partir de poblados del SNIT a escala 1:5 000 y modelo de interacción. 


\section{Conclusiones}

La realización de esta investigación pone en evidencia los factores que se relacionan directamente con la determinación del hábitat potencial del cocodrilo, tales como: la altitud menor a los $700 \mathrm{msnm}$, la presencia de áreas de manglar, de ríos caudalosos y anchos, y playones, así como de áreas de inundación. Estos factores se convierten en variables que demarcan su localización espacial dentro de un territorio. Con respecto a las condiciones socio ambientales en el Pacífico Central, durante los últimos años, han transformado el mosaico paisajístico en las partes más planas a una matriz más dominante por las actividades agropecuarias. Lo anterior sumado al acrecentamiento de la población humana, ha inducido la ocupación de nuevos espacios para las actividades antrópicas y el establecimiento de infraestructura en áreas consideradas óptimas para el cocodrilo, lo cual ha incrementado la interacción entre ambas especies, tal como se reflejó en las Zonas de interacción entre el ser humano y el cocodrilo abordados en este estudio.

Lo anterior es un proceso que debe de considerarse fundamental en la definición de estrategias, acciones y propuestas que deben ser promovidas desde el ordenamiento territorial en el Pacifico Central, las cuales son insuficientes o carecen de una aplicación óptima que garantice la protección del hábitat potencial del Crocodylus acutus, por lo que es fundamental incrementar actividades de planificación y ordenamiento territorial a nivel local, en conjunto con las instituciones asociadas al tema y de esa manera garantizar el desarrollo de las actividades antrópicas en las zonas que se consideran no aptas para la presencia del cocodrilo, con el fin de reducir la interacción entre el ser humano y la especie.

Lo antepuesto tiene que estar relacionado con estrategias de educación y sensibilización, los resultados obtenidos reflejan la necesidad de establecer programas de educación ambiental en las comunidades cercanas al hábitat potencial o áreas de transición entre hábitats, con el fin de dar a conocer las condiciones biológicas de la especie, donde se considere su importancia a nivel eco-sistémica y factores que afectan su hábitat aumentando la interacción entre el ser humano y el cocodrilo. Así como delimitar las áreas de accesibilidad por parte de las actividades turísticas, ya que constituyen una interacción directa con la especie, donde se generan imprudencias como ofrecer alimento, generando en ocasiones interacciones negativas. Y finalmente, sería importante para futuras investigaciones complementar este tipo de trabajo de forma multidisciplinaria, donde se logre incorporar y desarrollar el componente biológico, en asociación con las características geográficas que conforman su entorno natural.

\section{Bibliografía}

Andrade, A. (2013). Recomendaciones sobre la presencia del cocodrilo de río: Crocodylus acutus en cuerpos de agua del Campo de Golf "El Tigre” Nuevo Vallarta, 
Nayarit, Dirección estatal de Protección Civil y Bomberos División Fauna Silvestre, México.

Ambrosio, G., González, J. y Arévalo, V. (s.f.). Corrección radiométrica y geométrica de imágenes para la detección de cambios en una serie temporal, Departamento de Ingeniería de Sistemas y Automática, Universidad de Málaga.

Astorga, Y. (s.f.). "Situación del recurso hídrico", Décimo cuarto informe de la nación en desarrollo humano sostenible, CONARE, Costa Rica.

Bolaños, J., Sánchez, J. y Piedra, L. (1995). "Inventario y estructura poblacional de crocodílidos en tres zonas de Costa Rica. Laboratorio de Manglares, Área de Ecología y Manejo de Recursos Costeros", Rev. BioL Trop., 44(3)/45(1):283-287, 1996-1997, Universidad Nacional, Heredia, Costa Rica.

Bolaños, J. (2012). "Manejo de cocodrilos (Crocodylus acutus) en estanques de cultivo de tilapia en Cañas, Guanacaste", Revista de Ciencias Ambientales 43(1):6372. EISSN: 2215-3896.

- (2011). Propuesta plan de contingencia en el manejo de la población de cocodrilos del Gran Humedal del Tempisque (GHT), Asociación de Especialistas en Crocodílidos, Centroamérica, San José, Costa Rica.

(2002). Reflexiones acerca del conflicto entre el hombre (Homo sapiens) y el cocodrilo (Crocodylus acutus), Asociación de Especialistas en Crocodílidos, Centroamérica, San José, Costa Rica.

Cortijo, M. (s.f.). El cocodrilo de tumbres, Universidad Peruana de Ciencias Aplicadas (UPC), Perú. Recuperado de <http://hdl.handle.net/10757/345938>.

ESRI (2016). ArcGis (Spacial Analysis. Hydrology), EE.UU. Recuperado el 25 de abril del 2017 en la web: $<\mathrm{http}: / /$ resources.arcgis.com/search/?do=search\&start=0\&lg=en\&product=\&ver sion $=\&$ collection $=\&$ subject $=\& \mathrm{q}=$ hydrology $>$

- (2015). Comprender el análisis de distancia euclidiana, EE.UU. Recuperado el 20 de octubre del 2016 en la web: $<$ http://desktop.arcgis.com/es/arcmap/10.3/tools/spatial-analysttoolbox/understanding-euclidean-distance-analysis.htm>.

Estado de la Nación. (2008). "La zona marino-costera", Decimocuarto informe estado de la nación en desarrollo humano sostenible, CONARE, Costa Rica.

Fierro, J. (2010). Análisis estadístico univariado, bivariado y variables control, Universidad de Chile, Chile.

IMN (s.f.). Regiones y subregiones climáticas de Costa Rica, San José, Costa Rica.

García, J. y Buenrostro, A. (2015). Áreas de interacción entre humanos y cocodrilos (Crocodylus acutus Cuvier) en Chacahua, Oaxaca, México - Areas of Interaction Between Humans and Crocodiles (Crocodylus acutus Cuvier) in Chacahua, Oaxaca—,Editorial del Colegio de Postgraduados, Montecillo, Estado de México, México. 
Magaña, F., Delgado. A., Núñez, C., Campos, E. y Pecero, L. (2010). Ataques de cocodrilo de río (Crocodylus acutus) en Puerto Vallarta, Jalisco, México: presentación de cinco casos, Universidad de Guadalajara, México.

Morera, C. y Sandoval, L. (2015). Evaluación de los procesos de conectividad/fragmentación en Costa Rica en el año 2000, Escuela de Ciencias Geográficas, UNA, Heredia, Costa Rica.

Ramírez, M., Jiménez, M. y Martínez, A. (2005). "Estructura y densidad de la red de caminos en la Reserva de la Biosfera Mariposa Monarca”, Investigaciones Geográficas, Boletín (57):68-80, Instituto de Geografía, UNAM.

Sánchez, J. (2001). Estado de la población de cocodrilos (Crocodylus acutus) en el río Tempisque, Guanacaste, Costa Rica. INBIO, Heredia, Costa Rica.

Sandoval, L., Morera, C. y Sandoval, I. (2019). Fragmentación del hábitat del Cocodrilo Americano (Crocodylus Acutus: Reptilia: Crocodilidae) por actividades humanas en el Pacífico Central, de Costa Rica. Capítulo de libro. Paisaje y corredores en Costa Rica. Artículo en preparación.

Sandoval, I. (comunicación personal, 3 de noviembre, 2016). Distribución del cocodrilo durante la época lluviosa, Heredia, Heredia, Costa Rica.

Sandoval, I. y Bolaños, J. (2016). Análisis del Hábitat del Cocodrilo -Crocodylus acutus- (Reptilia: Crocodylidae) en el Gran Humedal del Tempisque Guanacaste y la vertiente del Pacífico Central de Costa Rica, Costa Rica, San José.

SINAC (2014). Sistema Nacional de Áreas de Conservación, San José, Costa Rica.

Submitted, T., Fulfillment, P., and Kong, H. (2010). Application of Urban Climatic Map to Urban Planning of High Density Cities - A Case Study of Hong Kong, China.

Santillán, R. (2013). Aplicación de Sistemas de Información Geográfica (SIG) para la elaboración de modelos zoogeográficos: un estudio de caso, tesis de maestría en Geomática, con mención en ordenamiento territorial, Universidad del Azuay, Cuenca, Ecuador. 